\title{
Interference Effects between Auger and Photoelectron Studied by Subnatural Linewidth Auger-Photoelectron Coincidence Spectroscopy
}

\author{
J. Viefhaus, ${ }^{1, *}$ G. Snell, ${ }^{1,2}$ R. Hentges,${ }^{1}$ M. Wiedenhöft, ${ }^{1}$ F. Heiser, ${ }^{1}$ O. Geßner, ${ }^{1}$ and U. Becker ${ }^{1}$ \\ ${ }^{1}$ Fritz-Haber-Institut der Max-Planck-Gesellschaft, Faradayweg 4-6, D-14195 Berlin, Germany \\ ${ }^{2}$ Fakultät für Physik, Universität Bielefeld, Universitätsstraße 25, D-33615 Bielefeld, Germany
}

(Received 6 October 1997)

\begin{abstract}
Using subnatural linewidth Auger-photoelectron coincidence spectroscopy following linearly polarized synchrotron radiation excitation we have measured the triply differential double photoionization cross section of xenon at a photon energy of $97.5 \mathrm{eV}$. At this photon energy the kinetic energy of the $4 d_{5 / 2}$ photoelectron line matches exactly the position of the ${ }^{1} S_{0} N_{5} O_{2,3} O_{2,3}$ Auger line and therefore the two electrons are indistinguishable. If the two electrons are emitted back to back in the plane of polarization the triply differential cross section fully vanishes. [S0031-9007(98)05406-4]
\end{abstract}

PACS numbers: 32.80.Hd, 07.81.+a, 32.80.Fb

Auger spectroscopy is a widely applied tool for the study of the electronic structure of gaseous and condensed matter as well as the chemical analysis of surfaces [1]. Its inherent limitation, however, is in the core hole lifetime width which limits the possible resolution of Auger electron lines. This inhibits in many cases the analysis of closely lying spectral features such as the vibrational structure in molecules. In the case of a resonant excitation the so-called Auger resonant Raman [2] effect can be used to overcome this problem by the utilization of a suitable narrow bandpass excitation source which is typically highly monochromatized synchrotron radiation. This method exploits the fact that the excitation and decay can be described only by a one-step process in which the intermediate core hole state only acts as an intermediate scattering state. Thus the observed linewidth in the Auger spectrum is limited only by the lifetime width of the final state which is orders of magnitudes smaller than the core hole lifetime width. Recently, many resonant Auger spectra with unprecedented resolution have been recorded this way for both atoms and molecules $[3,4]$. However, the method is closely linked to the existence of prominent core hole excited states below threshold where it gives detailed information on the electronic structure of highly excited singly charged ionic states. Therefore it cannot give direct insight to the electronic structure of the doubly charged ionic state which is formed above threshold. To overcome this limitation nonresonant subnatural linewidth Auger spectroscopy would be necessary. Such spectroscopy, however, requires in addition to the narrow excitation bandpass the coincident detection of the Auger and photoelectron with an energy resolution which is better than the lifetime width. This angle-resolved coincidence experiment results in the measurement of the triply differential cross section $d^{3} \sigma / d \Omega_{a} d \Omega_{b} d E_{a}$ (TDCS), where $\Omega_{a}$ and $\Omega_{b}$ are the angles of emission of the two electrons and $E_{a}$ is the energy of one of them. The energy of the other electron is defined by energy conservation $h \nu-I^{2+}=E_{a}+E_{b}$, where $h \nu$ is the photon energy and $I^{2+}$ is the double ionization potential of the particular final state of interest. In analogy to the method exploiting the Auger resonant Raman effect one might call such a measurement nonresonant Auger Raman spectroscopy.

In this Letter we report on the first measurement of a regular nonresonant Auger spectrum with an energy resolution below the lifetime width. We concentrate on a particular striking example for the coherent character of the two-electron states in a subnatural linewidth electronelectron coincidence experiment: we show that due to a destructive interference the triply differential photoionization cross section fully vanishes for Auger and photoelectrons under certain kinematic conditions.

Végh and Macek [5] predicted a strong interference effect if the two electrons have the same kinetic energy and are emitted back-to-back along the electric field vector. In this case the two-step model of the Auger decay is no longer valid because the two electrons are in coherent superposition of energy eigenstates. Depending on the multiplet character of the two-electron continuum wave function which has then to be used for the description of the photoionization process, the interference effect is either destructive or constructive. For the special case of the $\mathrm{Xe} \mathrm{N}_{5} \mathrm{O}_{2,3} \mathrm{O}_{2,3}$ Auger decay leading to the ${ }^{1} S_{0}$ doubly charged final state they concluded a strong reduction of the cross section due to its dominant singlet character. This is a consequence of a general selection rule [6] which is also responsible for the node observed in the triply differential cross section of the double photoionization of helium under the same kinematic conditions [7].

In a first attempt to observe the predicted interference Schwarzkopf and Schmidt [8] showed a reduction of the triply differential cross section of about one third compared to the case without any interference effect taken into account. Similar values were reported by Selles et al. [9] who also found an example of constructive interference of similar magnitude by studying a $\mathrm{Xe}^{2+}$ state of ${ }^{1} P^{o}$ symmetry. The differences with respect to the theoretical descriptions were attributed to the lack of total 
energy resolution rather than a different multiplet character of the two-electron continuum wave function. To reduce the problem of limited energy resolution similar studies were performed at lower photon energies in the case of so-called valence Auger decay of highly excited satellite states in neon [10]. Nevertheless, the theoretically predicted full annihilation of the triply differential cross section could not be observed.

In contrast to all previous investigations on this interference effect the present study utilized both high resolution in the photoexcitation as well as for the photoelectron spectrometry. The experiment was performed at the beam line BW3 of the Hamburger Synchrotronstrahlungslabor (HASYLAB) operating in the double bunch mode which allows timing experiments. The synchrotron radiation produced by an undulator is monochromatized by a modified SX-700 type plane grating monochromator [11]. In the photon energy range of interest the resolving power exceeds 5000 as the exit slit was set to less than $70 \mu \mathrm{m}$. This corresponds to a photon bandpass of less than $0.02 \mathrm{eV}$ at $97.5 \mathrm{eV}$ photon energy.

The experimental setup is depicted in Fig. 1. The incoming photon beam crosses an effusive Xe beam. During the experiment the Xe partial pressure was kept constant by a gas flow control system maintaining a chamber pressure of $2 \times 10^{-5} \mathrm{hPa}$. The emitted Auger and photoelectrons were detected in coincidence by a set of three time-of-flight electron analyzers, all of them lying in a plane perpendicular to the incoming photon beam. Two time-of-flight analyzers having a flight-path length of $0.46 \mathrm{~m}$ were positioned at angles $\theta_{1}=-54.7^{\circ}$ and $\theta_{2}=$ $180^{\circ}$ relative to the plane of linear polarization. The degree of linear polarization was determined to be $0.985(5)$ by measuring the angular distribution of the $\mathrm{Ne} 2 s$ and $2 p$ photoelectron lines [12]. The third analyzer mounted rotatable inside the vacuum chamber had a flight-path length of $0.14 \mathrm{~m}$. Its relative angle with respect to the plane of linear polarization was set to $\theta_{3}=0^{\circ}$. Consequently, three different relative emission angles, namely $\theta_{a b}=54.7^{\circ}, 125.3^{\circ}$, and $180^{\circ}$ could be investigated si-

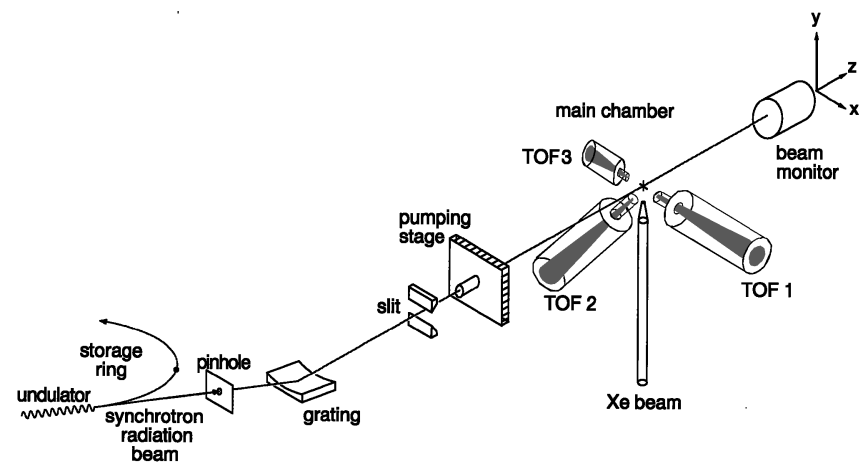

FIG. 1. Schematic setup of the time-of-flight (TOF) spectrometers used in the coincidence experiment following synchrotron radiation innershell excitation. multaneously. The relative energy resolution of the analyzers is $\approx 1.5 \%$ for the two longer analyzers and $\approx 4 \%$ for the shorter analyzer. The absolute energy resolution of the analyzers was enhanced by the use of a negative retardation potential inside the drift tube of the analyzers. Depending on the final kinetic energy the absolute energy resolution was in the range of 0.030 to $0.100 \mathrm{eV}$ for the longer analyzers and 0.080 to $0.250 \mathrm{eV}$ for the shorter one. The total coincidence resolution $\Delta E_{\text {coinc }}$ can then be derived from the energy resolution of the two electron analyzers $\Delta E_{a}, \Delta E_{b}$ and the photon bandwidth $\Delta E_{h \nu}$ using the formula

$$
\Delta E_{\mathrm{coinc}} \approx \sqrt{\left(\Delta E_{a}\right)^{2}+\left(\Delta E_{b}\right)^{2}+\left(\Delta E_{h \nu}\right)^{2}} .
$$

This setup makes it possible to investigate doubly charged final states with an energy resolution below the lifetime width. Figure 2 shows the first experimental proof for such a spectrum. Because of the fact that here both analyzers with better resolution contribute to the coincidence spectrum the undistorted ${ }^{1} D_{2}$ final state has a width of only $0.08(1) \mathrm{eV}$, which is significantly smaller than the $\mathrm{Xe} 4 d_{5 / 2}$ lifetime width of 0.121(4) eV [13].

In order to study the proposed interference effect rigorously it is important to tune the photon energy very carefully to the value where the two emitted electrons have equal kinetic energies. Using noncoincident spectra about $3 \mathrm{eV}$ below this photon energy, where the Xe $4 d_{5 / 2}$ photoelectron line does not overlap with the corresponding ${ }^{1} S_{0} N_{5} O_{2,3} O_{2,3}$ Auger line, the peak center of the Auger line was determined. The photon energy was then adjusted in a way that the $\mathrm{Xe} 4 d_{5 / 2}$ photoelectron line moved on top of the Auger line. Fine tuning of the photon energy was accomplished by monitoring on-line the

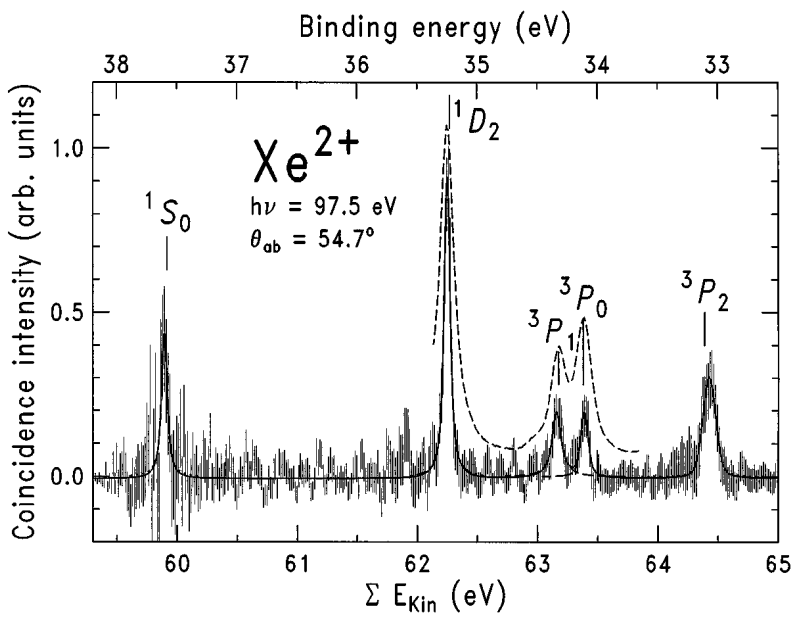

FIG. 2. $\mathrm{Xe}^{2+}$ spectrum: The coincidence intensity of TOF 1 and TOF 2 versus the sum of the kinetic energies of the two electrons is fitted by Voigt functions (solid line). The binding energies of the different doubly charged final states taken from optical spectroscopy [17] are indicated. For a comparison in resolution the dashed line represents the noncoincident Auger spectrum from TOF 1 . 
peak center of the sum of the two electron intensity contributions. In principle this procedure should give a very high precision in the order of $0.001 \mathrm{eV}$, but by performing frequent consistency checks during the coincidence measurement it was revealed that the long term stability of the photon energy was only $0.010 \mathrm{eV}$. The nature and the frequency of those drifts can be explained by photon beam shifts due to the heating of the monochromator optics as well as beam position instabilities of the storage ring itself. The absolute photon energy was calibrated using the energy positions of the $\mathrm{Xe} 4 d$ and $\mathrm{Kr} 3 d$ innershell resonances as references [14] which gave a value of $h \nu=97.496(10) \mathrm{eV}$. By taking into account the binding energy of the Xe $4 d_{5 / 2}$ photoelectron line [15] and the tabulated values of the corresponding doubly charged Xe states from optical spectroscopy $[16,17]$ a cross check value of $h \nu=97.501(13) \mathrm{eV}$ can be derived.

The total true coincidence count rate was about $0.6 \mathrm{~Hz}$ with a true-to-random ratio of $\approx 1 / 20$. In order to obtain significant results 15 spectra with a total collection time of $15000 \mathrm{~s}$ were taken. In the off-line data analysis the coincident spectra were corrected for random coincidences using an algorithm which takes into account the total count

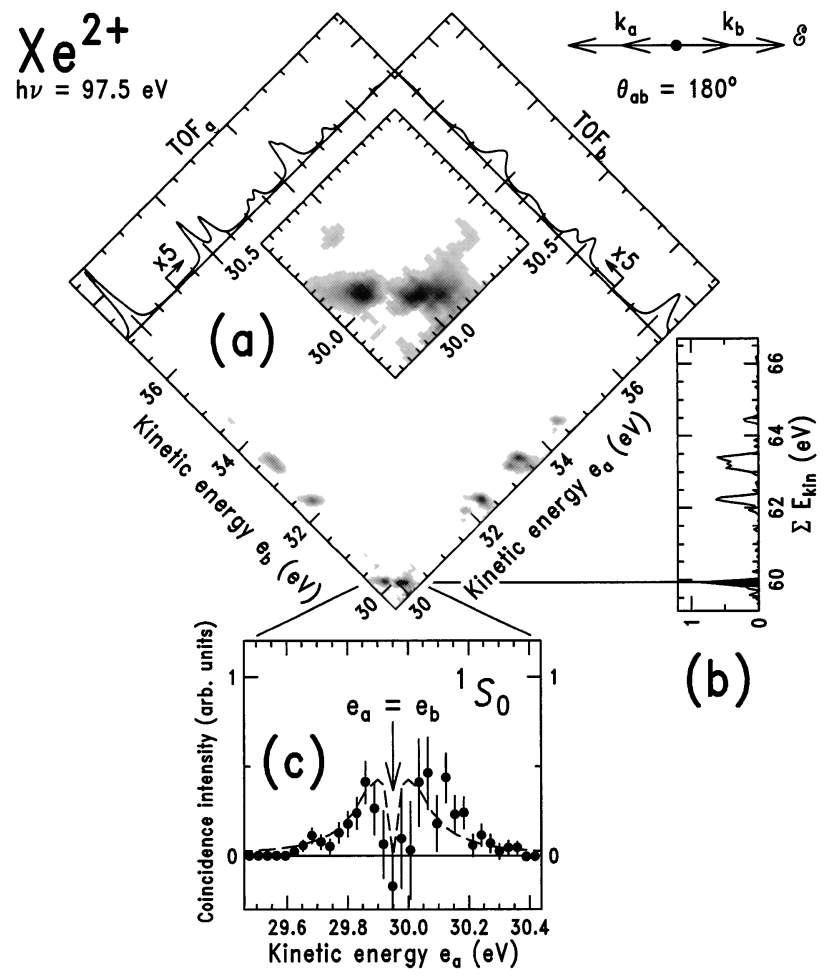

FIG. 3. $\mathrm{Xe}^{2+}$ spectra: (a) Two-dimensional coincidence map together with an enlarged inset of the interference region and the two noncoincident spectra of the corresponding electron analyzers, (b) integrated coincidence counts versus the sum of the kinetic energies of the two electrons and (c) the coincidence intensity of the ${ }^{1} S_{0}$ final state [dark shaded area in (b)] versus the kinetic energy of electron $e_{a}$. The broken curve is the scaled theoretical result of Sheinerman and Schmidt [19] (see text for details). rate, the simultaneously collected noncoincident electron spectra, and the dead times of the detectors. For a proper selection of the individual doubly charged final state the corrected coincidence spectrum as well as the two corresponding noncoincident spectra had to be converted from the time to the energy representation using the kinetic energy values of the Auger lines given by Aksela et al. [18] as references.

The obtained results for back-to-back emission of the two electrons are shown in Fig. 3. The corrected coincidence spectrum is the two-dimensional histogram which is plotted together with the corresponding two noncoincident spectra [Fig. 3(a)]. The di-cation spectrum results if the coincidence intensity is plotted versus the sum of the two electrons kinetic energies [Fig. 3(b)]. Here all doubly charged final states of the Xe $5 p^{-2}$ configuration are present. However, only the contributions via the $\mathrm{Xe} 4 d_{5 / 2}^{-1}$ intermediate states are visible as the $\mathrm{Xe} 4 d_{3 / 2}$ photoelectron line was retarded out of the spectrum. Consequently, the $\mathrm{N}_{4} \mathrm{O}_{2,3} \mathrm{O}_{2,3}$ Auger lines cannot contribute to the true coincidences, thereby proving that random coincidences have been properly taken into account. If one selects the $\mathrm{Xe} 5 p^{-2} \quad 1 S_{0}$ final state only [dark shaded area in Fig. 3(b)], the coincidence intensity of this particular state can be plotted versus the kinetic energy of one of the two electrons [Fig. 3(c)].

This intensity distribution shows a vanishing triply differential cross section for the case of exactly equal kinetic energies as proposed by Végh and Macek [5]. However, if the kinetic energies differ more than the lifetime width of the innershell hole, nonzero triply differential cross section can be found. This is in accord with the theoretical findings of Sheinerman and Schmidt [19] on the corresponding Auger transition of the beryllium atom. The broken curve in Fig. 3 was derived from their results by a

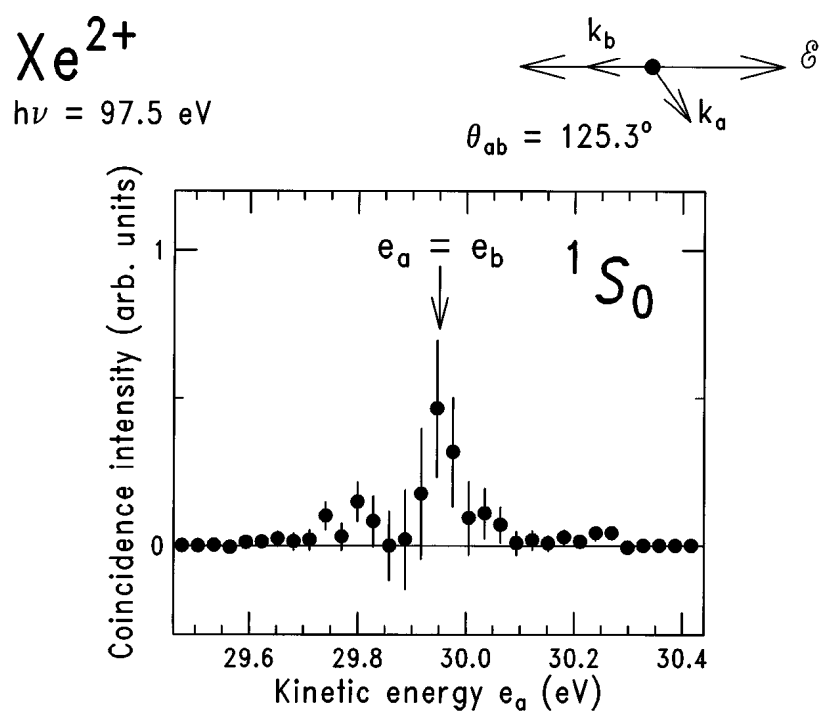

FIG. 4. $\mathrm{Xe}^{2+}$ spectra: same spectra as Fig. 3(c), but with different interelectron angle $\theta_{a b}=125.3^{\circ}$. 


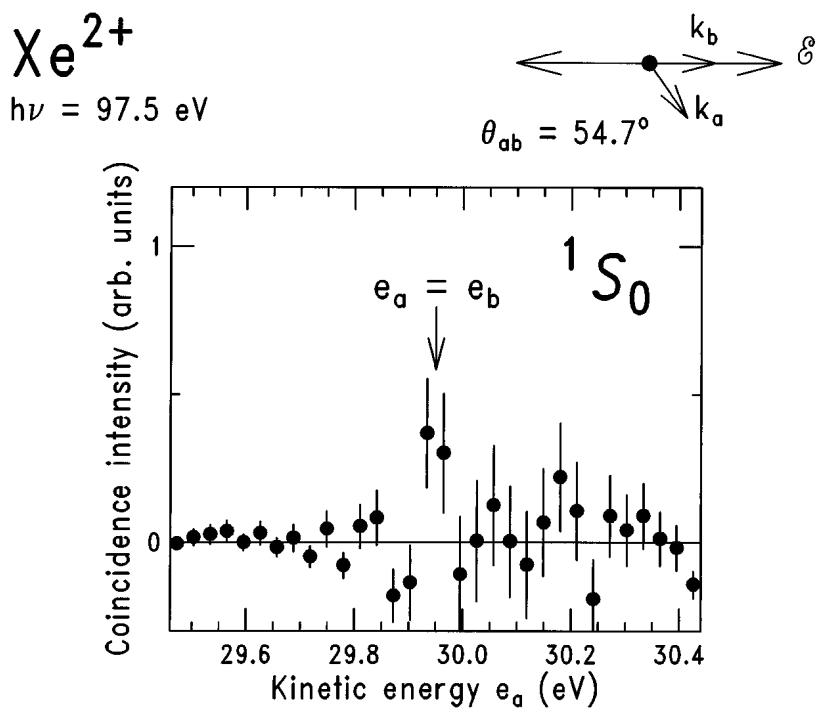

FIG. 5. $\mathrm{Xe}^{2+}$ spectra: same spectra as Fig. 3(c), but with different interelectron angle $\theta_{a b}=54.7^{\circ}$.

relative scaling factor with respect to our data and taking into account the fact that the lifetime width in our Xe case is roughly twice the width of their beryllium example.

The situation changes quite drastically if one compares these findings with the coincidence spectra recorded at the other two relative emission angles shown in Figs. 4 and 5. Here the triply differential cross section shows no dip at equal kinetic energies. Nevertheless, there are some hints for a superimposed interference structure although the comparatively low cross section at these angles in combination with the error bars due to random coincidences make a definitive judgment difficult.

Sheinerman and Schmidt [19] have shown that also at small relative emission angles strong interference should be observable. However, they restricted their study to examples having very small relative angles of $10^{\circ}$ and $5^{\circ}$, respectively, where the interference effects are getting much stronger again. Because of mechanical constraints the interelectron angle in our setup could not be made much smaller than the present value of $54.7^{\circ}$.

In conclusion, we have recorded the first nonresonant Auger spectrum with subnatural linewidth resolution using high-resolution time-of-flight electron-electron coincidence spectroscopy along with a narrow bandpass photoexcitation source. The corresponding data confirm the theoretical findings of Végh and Macek [5] that the triply differential cross section is fully annihilated in the $5 p^{-2}{ }^{1} S_{0}$ double ionization of $\mathrm{Xe}$ if the two electrons have exactly the same kinetic energy and are emitted back-to-back along the electric field vector. Further investigations corroborating the general relevance of this effect for other systems including the structure of vibrationally selected molecular Auger transition as well as the constructive interference for triplet coupled states are in preparation.

This work was supported in part by the Deutsche Forschungsgemeinschaft and the Bundesminister für Bildung, Wissenschaft, Forschung und Technologie. The support of the HASYLAB staff in particular by T. Möller is gratefully acknowledged.

*Electronic address: viefhaus@fhi-berlin.mpg.de

[1] W. Mehlhorn, Atomic Inner-Shell Physics, edited by B. Crasemann, in Physics of Atoms and Molecules (Plenum Press, New York, 1985), p. 119.

[2] G.S. Brown, M.H. Chen, B. Crasemann, and G. E. Ice, Phys. Rev. Lett. 45, 1937 (1980).

[3] A. Kivimäki, A. Naves de Brito, S. Aksela, H. Aksela, O.-P. Sairanen, A. Ausmees, S. J. Osborne, L. B. Dantas, and S. Svensson, Phys. Rev. Lett. 71, 4307 (1993).

[4] S. Svensson and A. Ausmees, Appl. Phys. 65, 107 (1997).

[5] L. Végh and J. H. Macek, Phys. Rev. A 50, 4031 (1994).

[6] F. Maulbetsch and J. S. Briggs, J. Phys. B 28, 551 (1995).

[7] O. Schwarzkopf, B. Krässig, J. Elminger, and V. Schmidt, Phys. Rev. Lett. 70, 3008 (1993).

[8] O. Schwarzkopf and V. Schmidt, J. Phys. B 29, 3023 (1996).

[9] P. Selles, J. Mazeau, P. Lablanquie, L. Malegat, and A. Huetz, J. Phys. B (to be published).

[10] S. J. Schaphorst, A. Jean, O. Schwarzkopf, P. Lablanquie, L. Andric, A. Huetz, J. Mazeau, and V. Schmidt, J. Phys. B 29, 1901 (1996).

[11] C. U.S. Larsson, A. Beutler, O. Björneholm, F. Federmann, U. Hahn, A. Rieck, S. Verbin, and T. Möller, Nucl. Instrum. Methods Phys. Res., Sect. A 337, 603 (1994).

[12] F. Wuilleumier and M. O. Krause, J. Electron Spectrosc. Relat. Phenom. 15, 15 (1979).

[13] A. Ausmees, S. J. Osborne, R. Moberg, S. Svensson, S. Aksela, O.-P. Sairanen, A. Kivimäki, A. Naves de Brito, E. Nõmmiste, J. Jauhiainen, and H. Aksela, Phys. Rev. A 51, 855 (1995).

[14] G. C. King, M. Tronc, F.H. Read, and R. C. Bradford, J. Phys. B 10, 2479 (1977).

[15] M. O. Krause, Synchrotron Radiation Research, edited by H. Winick and S. Doniach (Plenum Press, New York, 1980), p. 118.

[16] J. E. Hansen and W. Persson, Phys. Scr. 25, 487 (1982).

[17] W. Persson, C.-G. Wahlström, G. Bertuccelli, H. O. Di Rocco, J. G. Reyna Almandos, and M. Gallardo, Phys. Scr. 38, 347 (1988).

[18] H. Aksela, S. Aksela, and H. Pulkkinen, Phys. Rev. A 30, 865 (1984).

[19] S. A. Sheinerman and V. Schmidt, J. Phys. B 30, 1677 (1997). 